\title{
An alternative architecture and control strategy for hexapod positioning systems to simplify structural design and improve accuracy
}

\author{
Joseph H. Beno ${ }^{\mathrm{a}}$, John A. Booth ${ }^{\mathrm{b}}$, Jason Mock ${ }^{\mathrm{a}}$, \\ ${ }^{a}$ The University of Texas at Austin Center for Electromechanics, 1 University Station R7000, \\ Austin, TX 78712 \\ ${ }^{\mathrm{b}}$ The University of Texas at Austin McDonald Observatory, 1 University Station C1402, Austin, TX \\ 78712
}

\begin{abstract}
Hexapod systems (6 legged Stewart Platforms), offer advantages in accuracy over other positioning systems and are finding applications in numerous telescopes. However, instruments with increased sophistication for modern telescopes continue to grow in size and required positioning accuracy. This paper details an alternative hexapod configuration and design approach, particularly focused on relatively large, high precision hexapod systems supporting high mass payloads. The new configuration improves accuracy, reduces actuator mass, simplifies design, and reduces system cost but requires modest additional control algorithm sophistication.
\end{abstract}

Keywords: Hobby-Eberly, HET, HETDEX, Center for Electromechanics, large hexapod positioning systems, high payload hexapod positioning systems, high payload precision positioning systems, hexapod design and controls

\section{INTRODUCTION}

Conventional hexapod design focuses on optimizing design and placement of the six actuator-sensor units. Essentially, the actuators "define" the hexapod. For high mass payloads, maximum deflections occur in and around the powerful actuators, placing high emphasis on stiffness in and around actuators and their mounts (u-joints, spherical joints, etc.) This is particularly true for actuators that employ rotary sensors on shafts of screw or worm drives, a common topology because of complications associated with integrating true linear sensors into high force linear hexapod actuators. The alternative configuration and control system described in this paper decouples the sensor from the actuator. Linear sensors are positioned independent of actuator location, allowing independent optimization of actuator design/placement and sensor design/placement. Actuators are controlled with a force control strategy to yield precise sensor lengths. Truly optimized sensor design/placement yields improved correlation between sensor readings and payload configuration for improved accuracy with less reliance on compensation tables. The sensor "defines" the hexapod, substantially alleviating actuator stiffness requirements and reducing their mass and cost.

The hexapod system shown in Figure 1 is an example of the object of this paper. The six force actuators in Figure 1 are configured in a conventional arrangement, typical of many hexapod systems found across a wide assortment of applications, including high precision high payload telescope systems. These actuators can be configured in any stable arrangement devoid of singularities, as for any useful hexapod system. Note that the actuators are relatively slender, atypical of stiff actuators employed by hexapods with high precision, high accuracy, and large payload mass requirements. As will be described later, measuring length of these actuators is not relevant to hexapod controls, which greatly reduces their stiffness requirement and mass - these actuators are two force members that are controlled to output time dependant specified forces rather than specified lengths. Six linear displacement sensors are also shown in Figure 1. Note that these sensors are slender, as are their mounting hardware components. Although these sensors have high accuracy requirements, they bear negligible load so stiffness requirements can be met with minimal mass. As shown, the configuration and placement of these sensors meet certain requirements that are described later in the paper. The sensor configuration requirements, although quite flexible, are driven by control algorithm issues that are discussed later in the paper. Also note that Figure 1 shows that one plane contains upper joints for actuators and sensors and, similarly one 
plane contains lower joints for actuators and sensors. For the controls described in Section 3 of this paper, upper/lower sensor mounts must be in their respective planes. It is not necessary for actuator joints to also be in these planes, or even to be in planes at all, but it is more convenient.

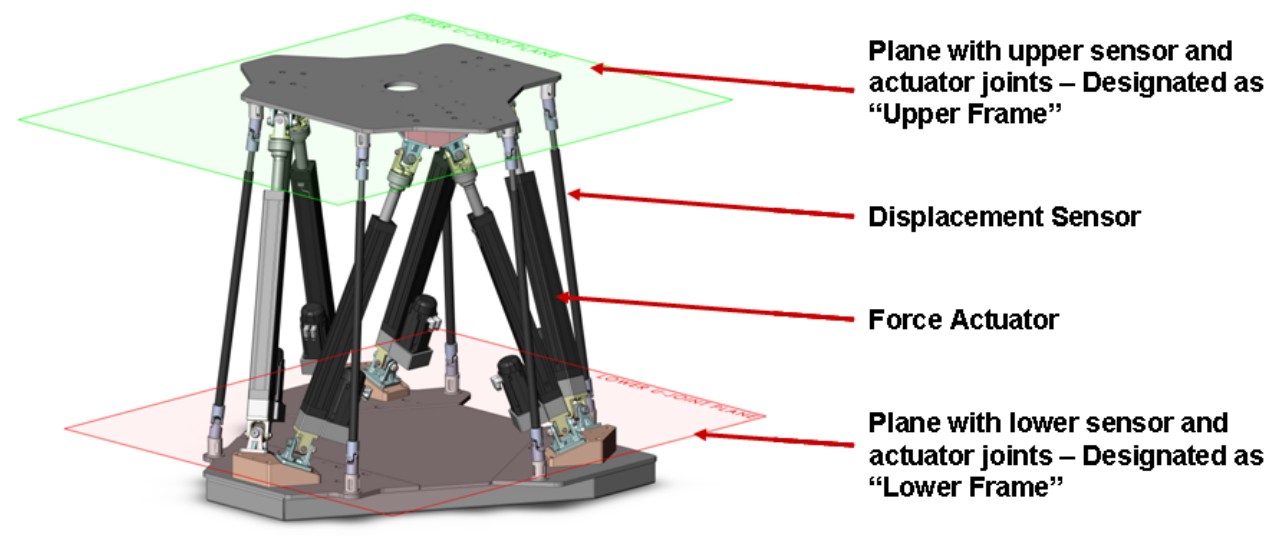

Figure 1. Hexapod design concept that separates sensor and actuator functions

The remainder of this paper is organized to first provide historical context and motivation for this hexapod design approach in Section 2. Section 3 provides in depth development of the analysis and control approach that enables this hexapod design approach. This is then followed by a brief discussion of sensor requirements (Section 4) and a very brief indication of benefits of the design approach (Section 5).

\section{HISTORICAL CONTEXT AND MOTIVATION FOR NEW DESIGN APPROACH}

\subsection{Historical Context in Astronomy}

The Stewart platform, or hexapod, has been used in a variety of applications since its invention in the 1950s, from flight simulators to spinal surgery platforms. It is commonly used today in large research telescopes to support and actively position secondary mirror assemblies to maintain optical alignment. When a large telescope is pointed at a sequence of different astronomical objects, which may appear anywhere from the zenith to near the horizon, the gravity vector on the primary mirror can change radically from one object to the next. The massive primary mirror can shift and tilt slightly in its mounting as a result, and the secondary end of the telescope can bend or sag slightly.

In older, optically slower telescopes built several decades ago, the effect of primary-secondary misalignment was less pronounced due to the much greater optical tolerances at large f/ratios. But for a modern primary mirror, which can have an f/ratio of 1.5 or less, primary-secondary misalignment can be a dominant source of misalignment and image quality degradation, and a means of positioning the secondary mirror in tip/tilt and the two lateral axes becomes necessary.

By using a hexapod mechanism to support the secondary mirror, combined with feedback from an optical wavefront sensor, small angular (e.g., tip and tilt) and lateral motions may be imparted to the mirror to maintain its optical alignment with the primary mirror. A hexapod has the additional advantage that it can position the secondary mirror about any arbitrary point in space. If the vertex of the secondary mirror is chosen as a rotation point, for instance, no lateral translation or "swing" of the mirror occurs when tip/tilt is adjusted, which was a problem for older trunnion designs. Active control of the secondary mirror to counteract the effects of a changing gravity vector also allows the telescope designer to minimize structural mass to support the sky end of the telescope, which can have structural stiffness and cost benefits as well. 
In the case of the Hobby-Eberly Telescope (HET) at McDonald Observatory in West Texas, the telescope uses a tracking device with a payload capacity of approximately $600 \mathrm{~kg}$ which incorporates a constantly moving hexapod to effect tip, tilt, and focus motions. The telescope differs from most astronomical research telescopes in that during an observation the primary mirror doesn't move, and stars are actively tracked using the tracking device. The telescope will soon be outfitted with a new tracker of much greater payload capacity (>5,000 kg), and which will incorporate a robust, high capacity hexapod to support and precisely position this large mass. During design of the new HET hexapod, the hexapod configuration described in this paper was conceived, albeit too late to be incorporated into the HET upgrade.

\subsection{Motivation for the New Design Approach}

Large scale hexapods suitable for new telescope designs typically consist of a drive screw and nut assembly serving as the final drive, with either the screw shaft itself or the nut being driven by a geared-down motor. The gearing is introduced to increase torque available to drive the hexapod leg, and to make the assembly self-locking in the powereddown condition. Because of the bulk of the hexapod leg and the often complicated and bulky end joints for precision hexapods that support large payloads, it can be difficult or impossible to integrate a precision linear encoder that attaches to actuator end joints and is near the centerline of the leg to reduce mechanical flexure not seen by the encoder. Consequently, rather than employing direct linear measurements, actuator length determination is often estimated from rotary encoders on the free end of the motor shaft. For many applications this may be good enough, but the information provided by the rotary encoder about the length of the hexapod extension will be degraded by the various interfaces between the encoder and the actual length change along the hexapod centerline. These will include coupling windup between the encoder and the motor, between the motor and the driven assembly, screw windup, play between the nut and the screw, and bearing and joint flexure throughout the strain path of the mechanical system. For high precision applications such as HET wide field upgrade and other large telescopes, this much motion, "unseen" by the encoder, can be well beyond typical tolerance requirements of a few microns. If using a rotary encoder, it is better to mount it directly on the final screw shaft, as this will eliminate/reduce many of the above sources of error. Nevertheless, estimating linear displacement can accomplished with higher accuracy by employing today's high performance linear sensors than by using rotary encoders that indirectly determine displacement estimates. The hexapod design approach described in this paper is motivated by a desire to enable simple mechanical integration of linear sensors into high precision, high accuracy hexapod systems that support heavy payloads.

Retrofit: There is another potential advantage to the concept presented here of linear encoders being geometrically independent of the hexapod force actuators, and that is in the case of an existing under-performing hexapod assembly. If the existing assembly has inadequate linear encoders, or rotary encoders but insufficient stiffness to achieve the desired overall performance, linear encoders could be added to the assembly in the manner described here to upgrade the device at considerably less cost than that of a complete assembly replacement.

\section{ENABLING CONTROLS APPROACH}

\subsection{Control Approach Overview}

Conventional approaches for controlling hexapod systems exploit actuator designs with imbedded/collocated sensors to determine actuator length (for example, see [1]. The sensed length is intended to be close to directly along the line of action of the actuator. For a desired pose of the upper hexapod frame relative to the lower frame (i.e., desired position and orientation of the upper frame with respect to the lower frame), desired hexapod actuator/leg lengths are easily determined with simple geometrical considerations. Deviations from the 6 desired leg lengths are read directly from the actuator imbedded sensor at each controller timestep to determine leg length errors. Simple Proportional-IntegralDerivative (PID) feedback controllers then seek to drive leg length errors to zero, thereby resulting in placing the upper frame in the desired pose. This process requires calculation of desired leg lengths for a given desired upper frame pose, a calculation that is referred to as the inverse kinematics problem. For this process, it is not necessary to actually determine pose of the upper frame relative to the lower frame from given leg lengths, a calculation that is labeled the forward kinematics problem.

It is well known that the forward kinematics solution for the general hexapod is very difficult to solve analytically and, depending on symmetry conditions for the hexapod configuration being analyzed, will generally have 8 to 40 [2,3] solutions. While some of these roots will be complex and some will represent unrealistic configurations (e.g., upper 
hexapod frame located below the lower hexapod frame), generally there will be multiple real and meaningful roots. This means that a given set of leg lengths will be associated with multiple possible poses. How does the PID control process described above ensure that driving the leg length errors to zero will place the upper frame in the desired pose rather than one of the other realistic possible poses? In practice this is not a problem for well-controlled hexapods. For wellcontrolled hexapods, in which leg-length errors are never allowed to grow unreasonably large during planned motions (e.g., tracking a trajectory), errors in pose of the upper frame do not overlap other realistic solutions.

For the conventional control approach described above, high accuracy hexapod systems require highly accurate measurement of leg lengths. For hexapod systems designed for high payloads, with sensors embedded/collocated with actuators so that the displacement measurement is close to being directly along the line of action of the actuator, compliance of the actuator, actuator joints, actuator mounting blocks and the upper and lower frame in the vicinity of the actuator mounting locations all compromise measurement accuracy. This leads to a requirement for a high degree of stiffness in these components. Furthermore, for displacement sensors in close proximity to the associated actuator, intended to remain parallel to the actuator so it accurately represents actuator length, additional errors such as alignment, can exacerbate the stiffness issue. Decoupling the sensor from the actuator by moving the sensor elsewhere on the hexapod system can alleviate the stiffness issue and improve system accuracy if properly accommodated in the control system. To achieve this objective, the control system must not need to know the length of the actuator, but must operate on the length of the sensors only. The sensors are light and present negligibly small loads to the hexapod compared to the payload loads. Consequently, designing sensor mounting and joint hardware that exhibits very small deflections does not require substantial masses. The general control strategy to accommodate the new sensor configuration will be described in the next paragraph and the remaining subsections in this controls section will develop the necessary math to enact the strategy.

The new control strategy treats the sensors as virtual ideal actuators with embedded displacement sensors. Displacement measurements for each of the six sensors are obtained at each controller timestep and compared with displacements associated with the desired pose of the upper frame to determine leg length errors, just as in the conventional hexapod control system. A PID feedback control algorithm then determines virtual forces required to drive errors toward zero, in the same manner as is done with the conventional hexapod control system. (Note that this process determines required force output from the virtual actuators. For the reader that tries to discriminate between "force control" and "position control," especially the reader that thinks about hexapod controllers as position control, please see the next paragraph). At this point, it is necessary to determine force outputs from the six real actuators that will produce the same effect on the upper frame as would be obtained from the six virtual actuators. This calculation relies on the forward kinematic solution (determining upper frame true pose from outputs from the six displacement sensors). Once the pose of the upper frame is known, the line of action of the six real actuators can be determined from their known upper and lower joint locations on the upper and lower frames. Then a linear set of six equations for the six unknown real actuator force outputs can be obtained by summing and comparing virtual forces and moments to forces and moments developed by the real actuator forces (real actuator forces are unknowns in the system of equations). Inverting this linear system of equations produces the required force output from the real actuators for the current timestep. Details are presented in the following sections.

Some readers may think that hexapods are being used to position the payload at the desired pose and, therefore, refer to their PID controller as a position controller. However, most hexapod systems, especially those designed for large payloads employ conventional rotary or linear actuators, often rotary permanent magnet motors driven by servoamplifiers and acting through a gear system or screw to produce linear motion. In these systems, servo-amplifiers output specified currents to actuator motors, which in turn create torques across the air gap (i.e., the gap between the rotor and stator). The hexapod PID controller outputs fully graduated current commands to the servo-amplifiers. Unless the actuators specifically employ stepper motors (uncommon for high force actuators), actuator output is ultimately achieved via current control through the servoamp, which is the equivalent of torque control on the motor and effectively force control on the actuator (for example, see [1]).

\subsection{Forward Kinematics I: Determining Angular Orientation of Upper Frame From The Six Displacement Sensors}

Attesting to the difficulty in solving the forward kinematics problem, a literature survey did not find any analytical solutions for the completely general hexapod configuration. Even several typical configurations, such as those used 
in many flight simulators, or the hexapod system design for the Hobby Eberly Telescope (HET) or the HET Wide Field Upgrade do not have known analytical solutions. Consequently, options are to rely on numerical solutions or choose a configuration for the displacement sensors (the virtual actuators) with known analytical solutions. Numerical solutions for the general hexapod configuration requires solution of six simultaneous non-linear equations. For large scale hexapods with large payloads, the upper and lower frame are typically separated by a meter or more and desired accuracy for telescope applications are a few microns or less. Intuition, confirmed by numerical experimentation, resulted in the conclusion that it is not realistic to expect adequate accuracy from numerical solutions of the general hexapod forward kinematic problem with relevant dimensions spanning 6 orders of magnitude or more. Again relying on literature surveys, the most useful sensor (virtual actuator) configuration and solution approach was described by $\mathrm{Ji}$ and $\mathrm{Wu}$ [2]. A general observation from the literature search is that we were not able to replicate the solution techniques described by many journal articles and we also found it necessary to modify the approach by Ji and Wu in order to develop a computationally reliable approach. Nevertheless, the formulation from Ji and Wu serves as the basis for this section.

We start by summarizing problem setup from Ji and Wu [2]. Figure 2, reproduced from Ji and Wu, describe (virtual) actuator joint locations on the lower frame (B1-B6) in coordinate system $\mathrm{O}(\mathrm{X}, \mathrm{Y}, \mathrm{Z})$ and joint locations on the upper frame (A1-A6) in coordinate system $O^{\prime}\left(X^{\prime}, Y^{\prime}, Z^{\prime}\right)$. Although not necessary, for most practical hexapods origins $\mathrm{O}$ and $\mathrm{O}^{\prime}$ are at the geometric center of the lower and upper joint locations, respectively. Origins $\mathrm{O}$ and $\mathrm{O}^{\prime}$ are connected by position vector $\mathrm{P}$, as shown. The hexapod configuration addressed by Ji and Wu and in this paper are limited to systems such that the coordinates ( $\mathrm{X}^{\prime}, \mathrm{Y}^{\prime}$ ) for joints A1-A6 are related by a positive real constant, $\mu$, to the coordinates $(\mathrm{X}, \mathrm{Y})$ for joints B1-B6 when the upper and lower frames are parallel and $\mathrm{O}$ is directly over O' (e.g., $\mathrm{P}=[0,0, \mathrm{Z}])$. Furthermore, upper and lower joints are required to be in individual upper and lower planes respectively.

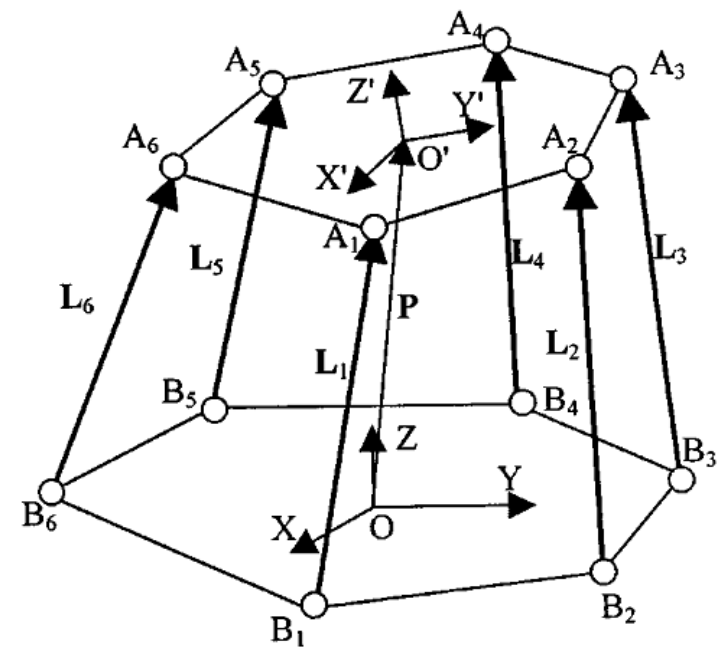

Figure 2. Hexapod configuration and variables

Consequently, representing upper joint locations by vectors in $\mathrm{O}^{\prime}$ as $\mathbf{A}_{\boldsymbol{n}}$ and lower joint locations by vectors in $\mathrm{O}$ as $\mathbf{B}_{n}$ for $n=1,2,3,4,5,6$; we have the following:

$$
\begin{aligned}
& \mathbf{A}_{\mathrm{n}}=\mu \mathbf{B}_{\mathrm{n}} \\
& \mathbf{B}_{\mathrm{n}}=\mathrm{B}_{\mathrm{x}} \mathbf{i}+\mathrm{B}_{\mathrm{y}} \mathbf{j}+0 \mathbf{k} \\
& \mathbf{A}_{\mathrm{n}}=\mathrm{A}_{\mathrm{x}} \mathbf{i}^{\prime}+\mathrm{Z}_{\mathrm{y}} \mathbf{j}^{\prime}=\mu\left(\mathrm{B}_{\mathrm{x}} \mathbf{i}^{\prime}+\mathrm{B}_{\mathrm{y}} \mathbf{j}^{\prime}\right)
\end{aligned}
$$

where $\mathbf{i}, \mathbf{j}, \mathbf{k}$ and $\mathbf{i}$, j’, $\mathbf{k}^{\prime}$ represent unit vectors in $\mathrm{O}$ or $\mathrm{O}^{\prime}$, respectively.

The vectors representing each leg, $L_{n}$ can be formed as:

$$
\mathbf{L}_{\mathrm{n}}=\mathbf{R} \mathbf{A}_{\mathrm{n}}+\mathbf{P}-\mathbf{B}_{\mathrm{n}}=(\mu \mathbf{R}-\mathbf{I}) \mathbf{B}_{\mathrm{n}}+\mathbf{P}
$$


where $\mathbf{R}$ is a rotation matrix (also called the direction cosine matrix) and $\mathbf{I}$ is the identity matrix. There are many choices for the rotation matrix and this topic will be discussed later. In the forward kinematics solution, the leg lengths are given, $\mathbf{B}_{\mathrm{n}}$ are known, and $\mathbf{R}$ and $\mathbf{P}$ must be determined. An equation for leg lengths becomes:

$$
\mathrm{L}_{\mathrm{n}}^{2}=\left[(\mu \mathbf{R}-\mathbf{I}) \mathbf{B}_{\mathrm{n}}+\mathbf{P}\right]^{\mathrm{T}}\left[(\mu \mathbf{R}-\mathbf{I}) \mathbf{B}_{\mathrm{n}}+\mathbf{P}\right]
$$

where $\mathrm{T}$ designates transpose. Using equation 2, this can be formatted as:

$$
\begin{aligned}
\mathrm{L}_{\mathrm{n}}{ }^{2}=\mathbf{P}^{\mathrm{T}} \mathbf{P}+2 \mathrm{~B}_{\mathrm{x} \_\mathrm{n}}\left[\mathbf{i}^{\mathrm{T}}\left(\mu \mathbf{R}^{\mathrm{T}} \mathbf{P}-\mathbf{P}\right)\right]+2 \mathrm{~B}_{\mathrm{y} \_n}\left[\mathbf{j}^{\mathrm{T}}\left(\mu \mathbf{R}^{\mathrm{T}} \mathbf{P}-\mathbf{P}\right)\right] \\
-2 \mu\left[\mathrm{B}_{\mathrm{x} n}{ }^{2}\left(\mathbf{i}^{\mathrm{T}} \mathbf{R} \mathbf{i}\right)+\mathrm{B}_{\mathrm{x} \_n} \mathrm{~B}_{\mathrm{y}_{\_} n}\left(\mathbf{i}^{\mathrm{T}} \mathbf{R} \mathbf{j}+\mathbf{j}^{\mathrm{T}} \mathbf{R} \mathbf{i}\right)\right. \\
\left.+\mathrm{B}_{\mathrm{y}_{-} \mathrm{n}}{ }^{2}\left(\mathbf{j}^{\mathrm{T}} \mathbf{R} \mathbf{j}\right)\right]+\left(1+\mu^{2}\right)\left(\mathrm{B}_{\mathrm{x} \_\mathrm{n}}{ }^{2}+\mathrm{B}_{\mathrm{y}_{-} \mathrm{n}}{ }^{2}\right)
\end{aligned}
$$

for $\mathrm{n}=1,2,3,4,5,6$

Equation (6) can be formatted as:

$$
\mathbf{Q} \mathbf{W}=\mathbf{d}
$$

where $\mathbf{W}=[\mathrm{W} 1, \mathrm{~W} 2, \mathrm{~W} 3, \mathrm{~W} 4, \mathrm{~W} 5, \mathrm{~W} 6]^{\mathrm{T}}$ with

$$
\begin{aligned}
& \mathrm{W} 1=\mathbf{P}^{\mathrm{T}} \mathbf{P} \\
& \mathrm{W} 2=\mathbf{i}^{\mathrm{T}}\left(\mu \mathbf{R}^{\mathrm{T}}-\mathbf{I}\right) \mathbf{P} \\
& \mathrm{W} 3=\mathbf{j}^{\mathrm{T}}\left(\mu \mathbf{R}^{\mathrm{T}}-\mathbf{I}\right) \mathbf{P} \\
& \mathrm{W} 4=\mathbf{i}^{\mathrm{T}} \mathbf{R} \mathbf{i} \\
& \mathrm{W} 5=\mathbf{i}^{\mathrm{T}} \mathbf{R} \mathbf{j}+\mathbf{j}^{\mathrm{T}} \mathbf{R} \mathbf{i} \\
& \mathrm{W} 6=\mathbf{j}^{\mathrm{T}} \mathbf{R} \mathbf{j}
\end{aligned}
$$

$\mathbf{Q}$ is a $6 \times 6$ matrix with rows $n=1,2,3,4,5,6$ as:

$$
\mathrm{Q}_{\mathrm{n}}=\left[1,2 \mathrm{~B}_{\mathrm{x} \_\mathrm{n}}, 2 \mathrm{~B}_{\mathrm{y}_{\_} \mathrm{n}},-2 \mu \mathrm{B}_{\mathrm{x} \_\mathrm{n}}{ }^{2},-2 \mu \mathrm{B}_{\mathrm{x} \_n} \mathrm{~B}_{\mathrm{y}_{\_} \mathrm{n}},-2 \mu \mathrm{B}_{\mathrm{y}_{\_} \mathrm{n}}{ }^{2}\right]
$$

and $\mathbf{d}=[\mathrm{d} 1, \mathrm{~d} 2, \mathrm{~d} 3, \mathrm{~d} 4, \mathrm{~d} 5, \mathrm{~d} 6]^{\mathrm{T}}$ with

$$
\mathrm{d}_{\mathrm{n}}=\mathrm{L}_{\mathrm{n}}{ }^{2}-\left(1+\mu^{2}\right)\left(\mathrm{B}_{\mathrm{x} \_\mathrm{n}}{ }^{2}+\mathrm{B}_{\mathrm{y} \_}{ }^{2}\right) \text { for } \mathrm{n}=1,2,3,4,5,6
$$

Equations (1) through (15) were obtained from Ji and Wu and then verified. Since $\mathbf{Q}$ and $\mathbf{d}$ consist entirely of known values, equation (7) can directly be inverted to obtain $\mathbf{W}$ as $\mathbf{Q}^{-1} \mathbf{d}$. Then $\mathbf{W}$ can be used to solve for the components of $\mathbf{R}$ and $\mathbf{P}$. Ji and Wu format $\mathrm{R}$ in terms of quaternions for this solution and present a numerical example so the reader can validate/compare results. However, the example used for validation by Ji and $\mathrm{Wu}$ is not of practical interest for hexapod configurations. Applying their solution to practical configurations resulted in complex results for quaternion components, which are not meaningful. Consequently, we developed an alternative approach for solving for $\mathbf{R}$ and $\mathbf{P}$, which is described below. The approach uses direct algebra and results in complicated rather long equations that are, nevertheless, easily handled by today's processors.

First a form of the rotation matrix is selected to represent the rotational pose of the upper frame. Euler developed 12 options with well-studied characteristics that represent net rotations as three successive rotations by angles in sequence of $\phi, \theta, \psi$. The 12 options relate these three angles to different combinations and sequences about axis X, Y, and Z. All twelve options have ambiguity arising from the second rotation. The options are categorized as type I or type II. Type I sequences have this ambiguity (singularity) for $\theta=+/$ - 90 degrees and type II exhibit the ambiguity at $\theta=0$ and 180 degrees [4]. Since hexapods typically operate with the upper frame between 0 and $+/$ - some angle less than 90 degrees, the type I Euler sequences are most relevant to avoid issues when $\theta$ is zero (e.g., when the upper frame is parallel to the lower frame). We arbitrarily selected the 1-3-2 sequence, also referred to as the X-Z-Y sequence. This means that the first rotation is through angle $\phi$ about the $\mathrm{X}$ axis; the second rotation is through angle $\theta$ about the new $\mathrm{Z}$ axis; and the 
third rotation is through angle $\psi$ about the final $\mathrm{Y}$ axis. Since our rotation operation represents an actual vector rotation (sometimes referred to as an alibi rotation) rather than a rotational change of the frame of reference for a vector fixed in space (an alias rotation), $\mathbf{R}$ can be obtained from Appendix E-2 of Wertz [4] by placing a negative sign in front of each angles shown. Consequently $\mathrm{R}$ is:

$$
\begin{aligned}
& \mathbf{R}=\left[\begin{array}{lll}
c \psi c \theta & -c \psi s \theta c \phi+s \psi s \phi & c \psi s \theta s \phi+s \psi c \phi
\end{array}\right. \\
& \mathrm{s} \theta \quad \mathrm{c} \theta \mathrm{c} \phi \quad-\mathrm{c} \theta \mathrm{s} \phi \\
& -s \psi c \theta \quad s \psi s \theta c \phi+c \psi s \phi \quad-s \psi s \theta s \phi+c \psi c \phi]
\end{aligned}
$$

where $\mathrm{c}=\cos$ and $\mathrm{s}=\sin$.

$\mathbf{R}$ has three unknowns: $\phi, \theta, \psi$. Equations (11) through (13) exclusively involve $\mathbf{R}$ without the vector $\mathbf{P}$ and can be used to develop 3 equations for the three unknown angles since an expression such as ( $\left.\mathbf{i}^{\mathrm{T}} \mathbf{R} \mathbf{i}\right)$ picks off an individual element of $\mathbf{R}$. Specifically, the 3 equations become:

$$
\begin{aligned}
& \mathrm{W} 4=\mathrm{R}_{11}=\mathrm{c} \psi \mathrm{c} \theta \\
& \mathrm{W} 5=\mathrm{R}_{12}+\mathrm{R}_{21}=-\mathrm{c} \psi \mathrm{s} \theta \mathrm{c} \phi+\mathrm{s} \psi \mathrm{s} \phi+\mathrm{s} \theta \\
& \mathrm{W} 6=\mathrm{R}_{22}=\mathrm{c} \theta \mathrm{c} \phi
\end{aligned}
$$

The algebra becomes less involved if the following substitutions are made (for these equations, $\mathrm{x} 1, \mathrm{x} 2$ and $\mathrm{x} 3$ are simply labels and have no relation to the $\mathrm{x}$ axis):

$$
\begin{aligned}
& \mathrm{c} \psi=\mathrm{x} 1 \quad \text { (then } \mathrm{s} \psi=\operatorname{sqrt}\left(1-\mathrm{x} 1^{2}\right) \\
& \mathrm{c} \theta=\mathrm{x} 2 \quad \text { (then } \mathrm{s} \theta=\operatorname{sqrt}\left(1-\mathrm{x}^{2}\right) \\
& \mathrm{c} \phi=\mathrm{x} 3 \quad \text { (then } \mathrm{s} \phi=\operatorname{sqrt}\left(1-\mathrm{x} 3^{2}\right)
\end{aligned}
$$

Consequently, equations (16) - (18) can be reformatted as:

$$
\begin{aligned}
& \mathrm{W} 4=\mathrm{x} 1 \mathrm{x} 2 \text { so } \mathrm{x} 2=\mathrm{W} 4 / \mathrm{x} 1 \\
& \mathrm{~W} 6=\mathrm{x} 2 \mathrm{x} 3 \text { so } \mathrm{x} 3=\mathrm{W} 6 / \mathrm{x} 2=\mathrm{W} 6 \mathrm{x} 1 / \mathrm{W} 4 \\
& \mathrm{~W} 5=-\mathrm{x} 1 \text { sqrt }\left(1-\mathrm{x} 2^{2}\right) \mathrm{x} 3+\operatorname{sqrt}\left(1-\mathrm{x}^{2}\right) \operatorname{sqrt}\left(1-\mathrm{x}^{2}\right)+\operatorname{sqrt}\left(1-\mathrm{x} 2^{2}\right)
\end{aligned}
$$

Equation (24) becomes:

$$
\begin{aligned}
& 0=-\mathrm{W} 5-\mathrm{x} 1 \operatorname{sqrt}\left(1-(\mathrm{W} 4 / \mathrm{x} 1)^{2}\right)(\mathrm{W} 6 \mathrm{x} 1 / \mathrm{W} 4) \\
& +\operatorname{sqrt}\left(1-\mathrm{x} 1^{2}\right) \operatorname{sqrt}\left(1-(\mathrm{W} 6 \mathrm{x} 1 / \mathrm{W} 4)^{2}\right)+\operatorname{sqrt}\left(1-(\mathrm{W} 4 / \mathrm{x} 1)^{2}\right)
\end{aligned}
$$

Equation (25) has one unknown, $\mathrm{x} 1$, and can be easily solved by a symbolic solver such as the solve function in MATLAB. The result is 8 solutions that are too long to include in this paper, but are easily cut and pasted into computer codes such as a MATLAB script file saved as a .m file. Once $\mathrm{x} 1$ is known, equations (22) and (23) determine $\mathrm{x} 2$ and $\mathrm{x} 3$ and then the arcos function determines $\phi, \theta, \psi$ and the entire rotation matrix follows. Of course, since $\cos (\psi)$ equals $\cos (-\psi)$, there are really 16 solutions to equation (25).

With 16 solutions for $\psi$, how can the "correct" solution be selected? This is really the same question we asked above with respect to the PID controller when we asked "How does the PID control process ensure that driving the leg length errors to zero will place the upper frame in the desired pose rather than one of the other realistic possible poses?” The answer now is essentially the same as the answer suggested with respect to the PID controller. Although multiple solutions of the 16 will be physically possible, if the hexapod is under good control the actual pose of the upper frame will be relatively close to the desired pose and, just like the PID controller, the "correct" solution will be the one closest to our desired pose.

\subsection{Forward Kinematics II: Determining Displacement Vector P From the Lower Frame To The Upper Frame From The Six Displacement Sensors}

Now that $\mathbf{R}$ is determined, the 3 unknown components of $\mathbf{P}=(\mathrm{x} \mathbf{i}+\mathrm{y} \mathbf{j}+\mathrm{z} \mathbf{k})$ can be determined from the three equations (8), (9), and (10). Again, although we had difficulties with the results from Ji and Wu [2], we found it 
convenient to use their nomenclature and pursue a straight-forward algebraic approach. Equations (8), (9), and (10) can be re-written as follows:

$$
\begin{aligned}
& x^{2}+y^{2}+z^{2}=W 1 \\
& U_{1} x+U_{2} y+U_{3} z=W 2 \\
& V_{1} x+V_{2} y+V_{3} z=W 3
\end{aligned}
$$

where $\mathrm{U}$ and $\mathrm{V}$ are defined by:

$$
\begin{aligned}
& \mathbf{U}=\mathbf{i}^{\mathrm{T}}\left(\mu \mathbf{R}^{\mathrm{T}}-\mathbf{I}\right) \\
& \mathbf{V}=\mathbf{j}^{\mathrm{T}}\left(\mu \mathbf{R}^{\mathrm{T}}-\mathbf{I}\right)
\end{aligned}
$$

First solving (27) and (28) simultaneously for $\mathrm{x}$ and $\mathrm{y}$ as a function of $\mathrm{z}$ and $\mathrm{W}$, and then substituting into (26) yields the following quadratic equation for $\mathrm{z}$ :

$$
0=z^{2}\left(B^{2}+D^{2}+1\right)+z(2 B C+2 D G)-W 1+C^{2}+G^{2}
$$

where

$$
\begin{aligned}
& \mathrm{B}=\left(\mathrm{U}_{3} \mathrm{~V}_{2}-\mathrm{U}_{2} \mathrm{~V}_{3}\right) / \mathrm{A} \\
& \mathrm{C}=\left(\mathrm{U}_{2} \mathrm{~W} 3-\mathrm{V}_{2} \mathrm{~W} 2\right) / \mathrm{A} \\
& \mathrm{D}=\left(\mathrm{U}_{1} \mathrm{~V}_{3}-\mathrm{U}_{3} \mathrm{~V}_{1}\right) / \mathrm{A} \\
& \mathrm{G}=\left(\mathrm{V}_{1} \mathrm{~W} 2-\mathrm{U}_{1} \mathrm{~W} 3\right) / \mathrm{A} \\
& \mathrm{A}=1 /\left(\mathrm{U}_{2} \mathrm{~V}_{1}-\mathrm{U}_{1} \mathrm{~V}_{2}\right)
\end{aligned}
$$

Once $z$ is determined, $x$ and $y$ are easily determined from the simultaneous solution of (27) and (28). Of course there are two solutions for $\mathbf{z}$ and therefore two possibilities for $\mathbf{P}$. It is easy to develop a set of rules that first seeks to eliminate a negative answer for $\mathrm{z}$ (which would put the upper frame below the upper frame) and, if both solutions for $\mathrm{z}$ are positive, select the $\mathbf{P}$ solution closest to the desired pose of the upper frame (again assuming good hexapod control).

\subsection{Determining Control Forces Required From Real (non-Virtual) Force Actuators}

Although Sections 3.2 and 3.3 seem rather involved, in reality the results are straight forward and suitable to integrate with the PID algorithms targeted for moderate controller hardware running at 500 to $1000 \mathrm{~Hz}$ (more than fast enough for good control of processes much more dynamic than telescope systems). With the results of Sections 3.2 and 3.3, the pose of the upper frame is known from the output of the six displacement sensors. Additionally, desired force output from the virtual actuators (collinear with the 6 displacement sensors) is determined from simple PID control algorithms acting on errors determined by comparing sensor output with desired sensor lengths ( $L_{n}$ for $\left.n=1,2,3,4,5,6\right)$. These PID control algorithms do not require the forward kinematic solutions developed in Sections 3.2 -3.3. However, now the force commands for the real actuators must be determined such that the real actuators effect the upper frame in the same manner as would the virtual actuators applying the forces specified by the PID control algorithms. For this determination, the line of action for the real actuators must be known and this requires the pose of the upper frame to be known as solved in sections 3.2-3.3.

The equations developed in this section refer to an origin centered on the lower frame, $\mathrm{O}(\mathrm{X}, \mathrm{Y}, \mathrm{Z})$. For this section the following nomenclature applies:

$\mathbf{F}_{\mathrm{v} \_\mathrm{n}}$ for $\mathrm{n}=1,2,3,4,5,6$ : Desired force output from virtual actuator ' $\mathrm{n}$ ' that is collinear with the displacement sensor ' $n$ '

$\mathbf{F}_{\mathrm{a} \_n}$ for $\mathrm{n}=1,2,3,4,5,6$ : Force output from real actuator ' $n$ '; the goal is for these actuators to have the equivalent result on the upper frame as would be obtained from the virtual actuators $\mathrm{F}_{\mathrm{v} \_\mathrm{n}}$.

$\mathbf{r}_{\mathrm{S} \_\mathrm{n}}$ for $\mathrm{n}=1,2,3,4,5,6$ : Vector from origin to upper frame joint for the sensor ' $\mathrm{n}$ ' (and virtual actuator ' $\mathrm{n}$ '); $\mathbf{r}_{\mathrm{s}-\mathrm{n}}=\mathbf{R} \mathbf{A}_{\mathrm{n}}+\mathbf{P}-\mathbf{B}_{\mathrm{n}}$

$\mathbf{r}_{\mathrm{a}_{\mathrm{n}} \mathrm{n}}$ for $\mathrm{n}=1,2,3,4,5,6$ : Vector from origin to upper frame joint for the real actuator ' $\mathrm{n}$ '

$\mathbf{u}_{s_{-} n}$ for $n=1,2,3,4,5,6$ : Unit vector along displacement sensor ' $n$ '; $\mathbf{u}_{s_{-} n}=u_{s_{-} n x} \mathbf{i}+u_{s_{-} n y} \mathbf{j}+u_{s_{-} n z} \mathbf{k}$

$\mathbf{u}_{a_{\_} n}$ for $n=1,2,3,4,5,6$ : Unit vector along real actuator ' $n$ '; $\mathbf{u}_{s_{-} n}=u_{a_{\_} n x} \mathbf{i}+u_{a_{\_} n y} \mathbf{j}+u_{a_{\_} n z} \mathbf{k}$ 
The process is a simple force and moment summation. For example summing forces in the $\mathrm{x}$ direction yields:

$$
\begin{aligned}
\mathrm{F}_{\mathrm{a} \_1} \mathrm{u}_{\mathrm{a} \_1 \mathrm{x}}+\mathrm{F}_{\mathrm{a} \_2} \mathrm{u}_{\mathrm{a} \_2 \mathrm{x}}+\mathrm{F}_{\mathrm{a} \_3} \mathrm{u}_{\mathrm{a} \_3 \mathrm{x}}+\mathrm{F}_{\mathrm{a} \_4} \mathrm{u}_{\mathrm{a} \_4 \mathrm{x}}+\mathrm{F}_{\mathrm{a} \_5} \mathrm{u}_{\mathrm{a} \_5 \mathrm{x}}+\mathrm{F}_{\mathrm{a} \_6} \mathrm{u}_{\mathrm{a} \_6 \mathrm{x}}= \\
\mathrm{F}_{\mathrm{v} \_1} \mathrm{u}_{\mathrm{s} \_1 \mathrm{x}}+\mathrm{F}_{\mathrm{v} \_2} \mathrm{u}_{\mathrm{s}_{2} 2 \mathrm{x}}+\mathrm{F}_{\mathrm{v} \_3} \mathrm{u}_{\mathrm{s} \_3 \mathrm{x}}+\mathrm{F}_{\mathrm{v} \_4} \mathrm{u}_{\mathrm{s}_{-} 4 \mathrm{x}}+\mathrm{F}_{\mathrm{v} \_5} \mathrm{u}_{\mathrm{s}_{-} 5 \mathrm{x}}+\mathrm{F}_{\mathrm{v} \_6} \mathrm{u}_{\mathrm{s} \_6 \mathrm{x}}
\end{aligned}
$$

with similar results for the $\mathrm{y}$ and $\mathrm{z}$ directions. Summing moments about the $\mathrm{x}$ axis yields equations of the form:

$$
\begin{aligned}
& \mathrm{F}_{\mathrm{a} \_1}\left(\mathrm{r}_{\mathrm{a} \_1 \mathrm{y}} \mathrm{u}_{\mathrm{a} \_1 \mathrm{z}}-\mathrm{r}_{\mathrm{a} \_1 \mathrm{z}} \mathrm{u}_{\mathrm{a} \_1 \mathrm{y}}\right)+\ldots+\mathrm{F}_{\mathrm{a} \_6}\left(\mathrm{r}_{\mathrm{a} \_6 \mathrm{y}} \mathrm{u}_{\mathrm{a} \_6 \mathrm{z}}-\mathrm{r}_{\mathrm{a} \_6 \mathrm{z}} \mathrm{u}_{\mathrm{a} \_6 \mathrm{y}}\right)= \\
& F_{v_{-} 1}\left(r_{s_{-} 1 y} u_{s_{-} 1 z}-r_{s_{-} 1 z} u_{s_{-} 1 y}\right)+\ldots+F_{v_{-} 6}\left(r_{s_{-} 6 y} u_{s_{-} 6 z}-r_{s_{-} 6 z} u_{s_{-} 6 y}\right)
\end{aligned}
$$

The result is 6 linear equations with six unknowns that can be configured as :

$$
\text { C } \mathbf{F}_{\mathrm{a}}=\mathbf{D}
$$

where $\mathbf{F}_{\mathrm{a}}=\left[\mathrm{F}_{\mathrm{a} \_1}, \mathrm{~F}_{\mathrm{a} \_2}, \mathrm{~F}_{\mathrm{a} \_3}, \mathrm{~F}_{\mathrm{a} \_4}, \mathrm{~F}_{\mathrm{a} \_5}, \mathrm{~F}_{\mathrm{a} \_6}\right]^{\mathrm{T}}$ and $\mathbf{C}$ is the matrix of coefficients on $\mathrm{F}_{\mathrm{a} \_n}$ from the left hand side of equations (32) and (33) and their counterparts in/about the ' $y$ ' and ' $z$ ' axis. $\mathbf{D}$ is a vector formed from the right hand side of equations (32) and (33) and their counterparts in/about the ' $y$ ' and ' $z$ ' axis. For example the first of the six components of $D$ is $\left(F_{v_{-} 1} u_{s_{-} 1 x}+F_{v_{-} 2} u_{s_{-} 2 x}+F_{v_{-} 3} u_{s_{-} 3 x}+F_{v_{-} 4} u_{s_{-} 4 x}+F_{v_{-} 5} u_{s_{-} 5 x}+F_{v_{-} 6} u_{s_{-} 6 x}\right)$. Equation (34) is quickly solved for the six unknown force magnitudes $\mathrm{F}_{\mathrm{a} \_n}$ by inverting $\mathbf{C}$.

\subsection{Hexapod Singularities and Other Considerations}

The hexapod configuration shown in Figures 1 and 2, illustrates the general concept and one configuration that has several advantages and one note of caution. First the note of caution.

As mentioned before, numerical solutions for the forward kinematics problem are not accurate enough for most telescope applications. In order to allow analytical solutions to the forward kinematic problem, we focused on a system such that the coordinates $\left(\mathrm{X}^{\prime}, \mathrm{Y}^{\prime}\right)$ for upper frame sensor joints A1-A6 are related by a positive real constant, $\mu$, to the coordinates $(\mathrm{X}, \mathrm{Y})$ for lower frame sensor joints B1-B6. However, any sensor configuration is acceptable that is either (1) analytically solvable or, (2) sufficiently symmetric that the non-linear numerical solution can be configured into a single equation (or very small number of equations) with a single unknown (or very small number of unknowns) that can be numerically solved with high accuracy. For the sensor configuration shown in Figure 2, the sensors need not be symmetrically or evenly distributed around the origins. In fact, care must be taken to ensure that the sensor joint patterns are not distributed on a circle (or other two-function quadratic curves) because the $\mathbf{Q}$ matrix, equation (14), is then singular [5]. The matrix condition number for $\mathbf{Q}$ serves as an indicator of how close $\mathbf{Q}$ is to being singular. For example, numerical experiments show that for the hexapod in Figure 2, if the sensor lower joints are offset from a circle by $0.5 \mathrm{~mm}$ in an alternating pattern (e.g., B1, B3, and B5 lies on one circle and B2, B4, B6 lie on another concentric circle with the differences in radii being $1 \mathrm{~mm}$ ) the condition of $\mathrm{Q}$ is adequate for good computational accuracy. Additionally, $\mu$, the constant relating the lower sensor joint pattern to the upper sensor joint pattern should not be 1 or too close to 1 . When $\mu$ is 1 , the sensors will be parallel, eliminating constraints represented by the 6 sensors to less than 6 and leading to singularities in the forward kinematics solution.

Now for some advantages. The primary advantages stem from design freedoms offered by sensor and actuator configurations, the cautions of the previous paragraph not withstanding. The fact that sensor upper joint pattern is a scaled version of lower sensor joint pattern still allows the sensors to be located with uneven spacing and nonsymmetrical patterns to exploit characteristics of the application or payload. Furthermore, the configuration of real actuators shown in Figure 1 is very common and versatile. Most importantly, optimization studies by Ma and Angeles [6] show this general configuration to have good stability characteristics. However, there is no requirement to use the actuator configuration shown in Figure 1. Any stable actuator configuration is acceptable.

\section{SENSOR CONSIDERATIONS}

The hexapod configuration and control approach presented in this paper is most beneficial for relatively large, high precision hexapod systems supporting high mass payloads. Implementation requires use of high precision external linear sensors and custom mounting system. The linear sensor and mounting system typically requires the following: 
- Absolute position feedback

- Micron accuracy and sub-micron resolution

- High-stiffness sensor mount

- Appropriate degrees of freedom in the integration and mounting scheme

Each linear sensor should be housed in an extendable structure (e.g., telescoping tube) and attached to the hexapod upper and lower frames with mounts that resemble typical hexapod actuator mounts, although on a much smaller scale. Typically the upper end of the sensor tube will be attached to the upper frame with a ball style joint (u-joint, heim joint, etc) and a rotary joint to allow necessary rotation about the sensor longitudinal axis. Similarly the other end of the sensor tube will be attached to the lower frame with a ball style joint, but there is not a need to include a rotary joint since the upper joint already allows this degree of freedom. Internally, one end of the telescoping tube will retain the body of the linear sensor while the other end attaches to the read head. The tube includes linear bearings to ensure that the read head properly maintains alignment and is not subjected to inappropriate loads. Bellows with purged dry air may also be needed.

Loads transmitted to/through the sensor upper and lower joints are very low since actuator force loads are managed elsewhere in the upper and lower frames. Consequently, although the joints for this custom sensor mount must be highly stiff, the mass can be small.

A suitable baseline sensor is the LC183 from Heidenhain corporation. Figure 3 shows a version of this sensor:

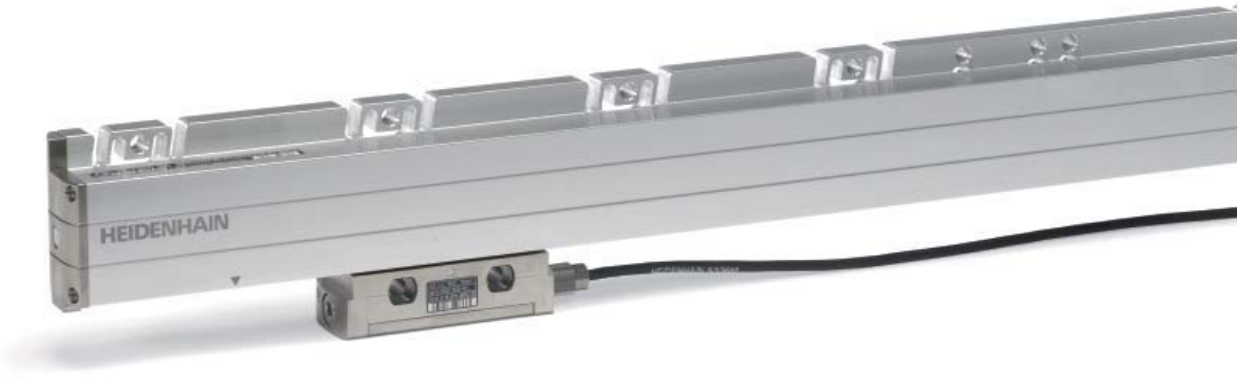

Figure 3. Heidenhain enclosed linear sensor

Specifications for the LC183:

- Accuracy grade: $+/-3$ microns

- Measuring Length: $140 \mathrm{~mm}$ to $4240 \mathrm{~mm}$

- Resolution: 0.005 microns

- $\quad$ Communication Protocol: EnDat 2.2

\section{BENEFITS OF NEW DESIGN APPROACH}

The primary benefit derived from this new design approach depends on what it is used for comparison. If compared against hexapod design approaches that use linear encoders within or directly adjacent to hexapod actuators to estimate actuator displacement for use in controls (typical of applications with low payload requirements), the new approach affords the designer greater freedom in the placement of hexapod sensors remote and independent from the actuator legs and likely results in improved control accuracy coupled with some mass reduction (especially in mounting hardware and joints). If compared against hexapod designs that employ rotary encoders on the final screw shaft or somewhere further back in the drive train to estimate actuator displacements, (typical of many applications with high payload requirements) then this approach can yield substantial actuator mass savings by reducing stiffness requirements, which reduces the necessary cross sectional area of load-bearing parts of the hexapod leg. This is because critical displacement information needed to effect accurate and precise hexapod motion is no longer dependant on actuator or joint stiffness. The real actuator's mission is simply to apply a specified force between its upper and lower mounts; controlling or 
knowing the length of the real actuators is not relevant. Actuator length is only relevant for virtual actuators, collocated with the sensor.

Preliminary assessments of potential hexapod actuator weight savings indicate that designing actuators based on material survival limits (e.g., no yield, adequate fatigue life, etc.) will likely allow actuator mass reduction of approximately $50 \%$ compared to actuators that also must be stiff enough to serve as a highly accurate, high resolution displacement sensors. This design approach will also likely meet requirements that actuator shape does not become so distorted under load that its line of action (defined as being between the actuator end mounts) adequately represents a two-force member. Alternatively, if stiffness concerns still exist but can be reduced by a factor of 2 compared to conventional (stiff) designs, actuator weight savings will be approximately $25 \%$. The $25 \%$ to $50 \%$ mass reductions result from things such as reducing end mount mass substantially and reducing size of actuator load-path components (e.g., roller screw and nut diameters, common components for high precision, high accuracy hexapod actuators).

\section{CONCLUSION}

A new method of configuring a hexapod positioning device has been developed that increases the design degrees of freedom with which the hexapod designer has to work. The method makes possible the physical separation of the hexapod linear encoders from the set of force actuators, allowing much greater geometric freedom in the placement of the linear encoders. In certain cases this can enable the use of linear encoders where it was previously impossible, and in other cases it can provide a means of achieving significant mass reduction by reducing the stiffness requirement associated with the use of rotary encoders. Finally, it may also allow inexpensive accuracy upgrades to existing hexapod systems.

\section{ACKNOWLEDGMENT}

Funding and motivation for this work was provided as part of the Hobby Eberly Telescope Wide Field Upgrade and the HET Dark Energy Experiment (HETDEX). HETDEX is led by the University of Texas at Austin McDonald Observatory and Department of Astronomy with participation from the Universitäts-Sternwarte of the LudwigMaximilians-Universität München, the Max-Planck-Institut für Extraterrestriche-Physik (MPE), Astrophysikalisches Institut Potsdam (AIP), Texas A\&M University, Pennsylvania State University, and the HET consortium. In addition to Institutional support, HETDEX is funded in part by gifts from Harold C. Simmons, Robert and Annie Graham, The Cynthia and George Mitchell Foundation, Louis and Julia Beecherl, Jim and Charlotte Finley, Bill and Bettye Nowlin, Robert and Fallon Vaughn, Eric Stumberg, and many others, by AFRL under agreement number FA9451-04-2-0355, and by the Texas Norman Hackerman Advanced Research Program under grants 003658-0005-2006 and 003658-02952007.

\section{REFERENCES}

[1] Zierer, J.J., Mock, J.R., Beno, J.H., Good, J. Booth, J.A., Lazzarini, P, Fumi, P. Anaclerio, E. “The Development of high-precision hexapod actuators for the Hobby-Eberle Telescope Wide Field Upgrade,” Proc. SPIE 7733-49 (2010)

[2] Ji, P., Wu, H. "A closed-form forward kinematics solution for the 6-6 ${ }^{\mathrm{P}}$ Stewart Platform.” IEEE Transactions on Robotics and Automation, Vol 17, No. 4, August 2001

[3] Gao, X-S, Lei, D., "Generalized stewart platforms and their direct kinematics," MM Research Preprints, 64-85, MMRC, AMSS, Academia, Sinica, Beijing, No, 22, December 2003

[4] Wertz, J.R., ed., “Spacecraft Attitude Determination and Control," Kluwer Academic Publishers, Boston, MA, and D. Reidel Publishing Company, Dordrecht, Holland, 1978

[5] Charters, T., Enguica, R., Freitas, P., "Detecting Singularities of Stewart Platforms," Mathematics-in-Industry Case Studies Journal, Volume 1, pp 66-80, 2009

[6] Ma, O., Angeles, J., “Optimum architecture and design of platform manipulators," IEEE Xplore, license to University of Texas at Austin, 1991 